\title{
Genetic variations in histidine-rich protein 2 and histidine-rich protein 3 of Myanmar Plasmodium falciparum isolates
}

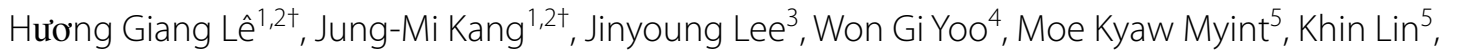

Tong-Soo Kim ${ }^{3 *}$ and Byoung-Kuk Na ${ }^{1,2^{*}}$

\begin{abstract}
Background: Malaria rapid diagnostic tests (RDTs) are precious tools to diagnose malaria. Most RDTs used currently are based on the detection of Plasmodium falciparum histidine-rich protein 2 (PfHRP2) in a patient's blood. However, concern has been raised in recent years that deletion of pfhrp2 in the parasite could affect the accuracy of PfHRP2based RDTs. In addition, genetic variation in pfhrp2 might influence the accuracy and sensitivity of RDTs. In this study, the genetic variation in pfhrp2 and pfhrp3 in Myanmar P. falciparum isolates was analysed.

Methods: Blood samples were collected from malaria patients who were infected with P.falciparum in Mandalay, Naung Cho, Tha Beik Kyin, and Pyin Oo Lwin, Upper Myanmar between 2013 and 2015. The pfhrp2 and pfhrp3 were amplified by nested polymerase chain reaction (PCR), cloned and sequenced. Genetic variation in Myanmar pfhrp2 and pfhrp3 was analysed using the DNASTAR program. Comparative analysis of Myanmar and global pfhrp2 and pfhrp3 isolates was also performed.
\end{abstract}

Results: One-hundred and two pfhrp2 and 89 pfhrp 3 were amplified from 105 blood samples, of which 84 pfhrp2 and 56 pfhrp3 sequences were obtained successfully. Myanmar pfhrp2 and pfhrp3 showed high levels of genetic variation with different arrangements of distinct repeat types, which further classified Myanmar pfhrp2 and pfhrp3 into 76 and 47 haplotypes, respectively. Novel amino acid changes were also found in Myanmar pfhrp2 and pfhrp3, but their frequencies were very low. Similar structural organization was shared by Myanmar and global pfhrp2 and pfhrp3, and differences in frequencies of repeat types and lengths were also observed between and among global isolates.

Conclusion: Length polymorphisms and amino acid substitutions generated extensive genetic variation in Myanmar pfhrp2 and pfhrp3. Comparative analysis revealed that global pfhrp2 and pfhrp3 share similar structural features, as well as extensive length polymorphisms and distinct organizations of repeat types. These results provide a better understanding of the genetic structure of pfhrp2 and pfhrp3 in global P. falciparum populations and suggest useful information to develop RDTs with improved quality.

Keywords: Plasmodium falciparum, Histidine-rich protein 2, Histidine-rich protein 3, Myanmar, Genetic polymorphism

\footnotetext{
*Correspondence: tongsookim@inha.ac.kr; bkna@gnu.ac.kr

${ }^{\dagger}$ Hương Giang Lê and Jung-Mi Kang contributed equally to this study

${ }^{1}$ Department of Parasitology and Tropical Medicine and Institute

of Health Sciences, Gyeongsang National University College of Medicine,

Jinju 52727, Republic of Korea

${ }^{3}$ Department of Tropical Medicine and Inha Research Institute

for Medical Sciences, Inha University College of Medicine, Incheon 22212,

Republic of Korea

Full list of author information is available at the end of the article
}

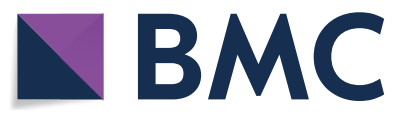

(c) The Author(s) 2020. This article is licensed under a Creative Commons Attribution 4.0 International License, which permits use, sharing, adaptation, distribution and reproduction in any medium or format, as long as you give appropriate credit to the original author(s) and the source, provide a link to the Creative Commons licence, and indicate if changes were made. The images or other third party material in this article are included in the article's Creative Commons licence, unless indicated otherwise in a credit line to the material. If material is not included in the article's Creative Commons licence and your intended use is not permitted by statutory regulation or exceeds the permitted use, you will need to obtain permission directly from the copyright holder. To view a copy of this licence, visit http://creativeco mmons.org/licenses/by/4.0/. The Creative Commons Public Domain Dedication waiver (http://creativecommons.org/publicdomain/ zero/1.0/) applies to the data made available in this article, unless otherwise stated in a credit line to the data. 


\section{Background}

Myanmar has the majority of malaria cases and deaths in Southeast Asia, but the incidence of the disease has declined dramatically during the last decade and is progressing steadily towards elimination [1]. The annual number of malaria cases in the country has dropped from approximately 700,000 in 2010 to about 85,000 in 2017 [1]. Microscopic examination of a blood smear is the primary diagnostic tool for malaria in Myanmar, but malaria rapid diagnostic tests (RDTs) have become a valuable alternative for use in remote areas where microscopy may not be feasible or where microscopy results would not be available immediately. RDTs offer a practical alternative to microscopy because they do not require a laboratory or special equipment, are simple to use, and provide reliable results in a short time [2,3]. RDTs have been introduced as reliable diagnostic tools in many malariaendemic areas, including Myanmar.

Malaria RDTs are designed to detect one or more Plasmodium antigens in a patient's blood by using specific monoclonal antibodies. Several antigens of Plasmodium falciparum have been utilized in RDTs for malaria detection, including histidine-rich protein 2 (PfHRP2), lactate dehydrogenase (PfLDH) and aldolase [4]. Among these antigens, PfHRP2 is the most widely employed in commercially available malaria RDTs at present, because of its abundant expression in the asexual blood stage of $P$. falciparum [5-7], structural stability [8] and high specificity recognized by multiple antibodies [9-11]. PfHRP2 is a protein encoded by pfhrp2, which is located in the subtelomeric region of chromosome 8 . This $P$. falciparumspecific protein is expressed abundantly in the infected erythrocyte surface of the blood stage and early gametocyte stage $[12,13]$. Some of the PfHRP2-based RDTs can cross-react with a structural homologue, $P$. falciparum histidine-rich protein 3 (PfHRP3) that is encoded by $p f h r p 3$ and shares high sequence identity and epitope similarity with PfHRP2 $[13,14]$. The $p f h r p 2$ and $p f h r p 3$ genes are predicted to be derived from a common ancestral gene. As such, these near-duplicate genes may compensate each other in function [7]. However, it has been reported recently that $P$. falciparum field isolates in some parts of malaria-endemic regions lack pfhrp2 [15-20]. Deletion of $p f h r p 2$ could affect the accuracy of PfHRP2based RDTs and lead to false-negative results followed by inappropriate treatment, which in turn causes negative impact for effective control and elimination of malaria. Furthermore, co-deletion of $p f h r p 2$ and $p f h r p 3$ has also been identified $[8,21]$. In addition to the density of the parasite or the lack of PfHRP2 expression, it has also been suggested that genetic diversity in $p f h r p 2$ and pfhrp 3 could affect the sensitivity of PfHRP-based RDTs
[22, 23]. Therefore, monitoring parasite factors that can affect performance of RDT-based diagnosis is important.

In this study, the genetic variation in pfhrp 2 and $p f h r p 3$ of Myanmar P. falciparum isolates was analysed. The diversity of the two genes from global $P$. falciparum isolates was also investigated comparatively to gain in-depth understanding of the genetic diversity and population structure of global $p f h r p 2$ and $p f h r p 3$.

\section{Methods}

\section{Study sites and blood sample collection}

A total 105 blood samples from malaria patients infected with P. falciparum were collected during a previous study conducted in Myanmar between 2013 and 2015 [24]. The patients were detected during regional malaria surveys, which were conducted in the regions of Naung Cho, Pyin Oo Lwin, Tha Beik Kyin, and Mandalay in Upper Myanmar (Additional file 1: Fig. S1). Malaria infection was diagnosed by microscopic analysis of thin and thick blood smears. Finger-prick blood was taken from P. falciparum-infected patients and spotted in Whatman 3MM filter (GE Healthcare, Maidstone, UK) for confirmation by polymerase chain reaction (PCR) targeting $18 \mathrm{~S}$ ribosomal RNA (rRNA) gene [24]. Informed consent was obtained from all patients before blood collection. The study protocol was approved by either the Ethics Committee of the Ministry of Health, Myanmar (97/Ethics 2015) or the Biomedical Research Ethics Review Board of Inha University School of Medicine, Republic of Korea (INHA 15-013).

\section{Genomic DNA extraction and amplifications of pfhrp2 and pfhrp3}

Genomic DNA was extracted from the dried blood spots using the QIAamp DNA Blood Kit (Qiagen, Hilden, Germany) following the manufacturer's instructions. The primers specific for $p f h r p 2$ and $p f h r p 3$ were designed and used (Additional file 2: Table S1). Both genes were amplified by nested PCR methods. Each PCR was done with thermal cycling conditions: $94{ }^{\circ} \mathrm{C}$ for $5 \mathrm{~min}$, and 35 cycles of $94{ }^{\circ} \mathrm{C}$ for $1 \mathrm{~min}, 53{ }^{\circ} \mathrm{C}$ for $1 \mathrm{~min}$, and $72{ }^{\circ} \mathrm{C}$ for $1.5 \mathrm{~min}$, followed by the final extension at $72{ }^{\circ} \mathrm{C}$ for $10 \mathrm{~min}$. In order to minimize the nucleotide mis-incorporation during amplification, Ex Taq DNA polymerase (Takara, Otsu, Japan), which has a proof-reading activity, was used in all PCR steps. Each PCR product was resolved on $1.5 \%$ agarose gel, extracted from the gel, and cloned into T\&A cloning vector (Real Biotech Corporation, Banqiao, Taiwan). Each ligation mixture was transformed into Escherichia coli DH5 $\alpha$ competent cells. Colony PCR was performed to select the positive clones with appropriate inserts. The nucleotide sequences of the cloned pfhrp 2 and $p f h r p 3$ were analysed by automatic DNA sequencing 
with M13 forward and M13 reverse primers by the Sanger method. Plasmids from at least two independent clones from each transformation mixture were sequenced to verify the sequence accuracy. The nucleotide sequences of Myanmar pfhrp2 and pfhrp3 analysed in this study have been deposited in the GenBank database under the accession numbers KX138275-KX138311, MG417056MG417080, and MT591418-MT591439 for pfhrp2 and KX138312-KX138340 and MT591440-MT591466 for pfhrp3.

\section{Sequence analyses of Myanmar and global pfhrp2 and pfhrp3}

The nucleotide and deduced amino acid sequences of Myanmar pfhrp 2 and pfhrp3 were analysed using EditSeq and SeqMan in the DNASTAR package (DNASTAR, Madison, WI, USA). Genetic variations of pfhrp 2 and pfhrp3 in global P. falciparum isolates were also analysed. The pfhrp 2 and pfhrp 3 sequences deposited in public database were used in this study. The pfhrp 2 sequences analysed in this study were from China, India, Sri Lanka, Thailand, Philippines, Cambodia, Vietnam, Central African Republic, Ghana, Haiti, Kenya, Madagascar, Nigeria, Tanzania, French Guinea, Brazil, Honduras, Papua New Guinea, Solomon Islands, and East Timor (Additional file 3: Table S2). For $p f h r p 3$, the sequences from Cambodia, India, Philippines, Kenya, Madagascar, Nigeria, Peru, Colombia, Papua New Guinea, and Solomon Islands were analysed (Additional file 4: Table S3).

\section{Results}

Amplification of pfhrp2 and pfhrp3 in Myanmar Plasmodium falciparum isolates

PCR amplification of pfhrp2 and pfhrp 3 from $105 P$. falciparum-infected blood samples resulted in successful amplification of 102 pfhrp 2 and 89 pfhrp3. The approximate sizes of amplified products were highly variable, ranging 100-1000 bp for pfhrp2 and 50-600 bp for $p f h r p 3$. Of these, 84 pfhrp2 and 56 ffhrp 3 PCR products were cloned successfully and sequenced for further assessments. The remaining 19 pfhrp 2 and 33 pfhrp 3 PCR products were excluded from this study because the quality of the amplicons was not adequate for further analysis, despite repeated attempts.

\section{Polymorphic character of Myanmar pfhrp2}

Seventy-six distinct haplotypes of $p f h r p 2$ were identified in 84 Myanmar P. falciparum isolates (Fig. 1). Each haplotype consisted of different numbers of repeats ranging from 3 to 36. Different arrangements of distinct repeat types resulted in size variation between and among haplotypes. Haplotype 13 (H13) showed the highest prevalence with $4.76 \%$ (4/84), followed by haplotype 55 (H55), accounting for $3.57 \%$ (3/84). Most Myanmar pfhrp2 started with 1-6 copies of type 1 repeat (AHHAHHVAD, 96.4\%) and terminated with type 12 repeat (AHHAAAHHEAATH, 94\%). However, three pfhrp 2 haplotypes (H23, H24, H76) began with type 2 repeat (AHHAHHAAD) and finished with type 12 repeat. Five haplotypes (H16, $\mathrm{H} 20, \mathrm{H} 21, \mathrm{H} 32, \mathrm{H} 48$ ) also started with type 1 repeat, but they terminated with type 4 (AHH), type 6 (AHHATD), or type 10 (AHHAAAHHATD).

\section{Polymorphic character of Myanmar pfhrp3}

Forty-seven distinct haplotypes of $p f h r p 3$ were observed in 56 Myanmar P. falciparum isolates (Fig. 2). Eleven different types of repeat were observed in Myanmar pfhrp3 and each haplotype was constructed with different numbers of the repeats ranging from 2 to 25 . Structural features of Myanmar pfhrp3 haplotypes were highly diverse, but all haplotypes shared similar patterns. Most haplotypes started with type 1 repeat (AHHAHHVAD) and terminated with type 4 repeat (AHH). Two exceptions were haplotypes 38 and 46. Haplotype 38 stared with type 1 repeat but finished with type 15 repeat (AHHAHHAAN). Haplotype 46 began with type 7 repeat (AHHAAD) and terminated with type 4 repeat (AHH). Non-repeat (NR) regions were scattered randomly in the sequences of most haplotypes. The length variation in Myanmar pfhrp3 was caused mainly by repeating numbers of type 16 (AHHAAN), type 17 (AHHADG), or type 18 (AHHDD).

\section{Amino acid changes in Myanmar pfhrp2 and pfhrp3}

Amino acid substitutions were identified in repeat types 1, 2, 6, and 7 of Myanmar pfhrp2 (Table 1). Six variants

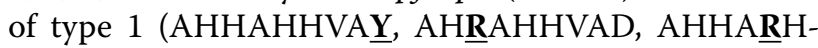
VAD, AHㅁAHVAD, AHHAHHEAD, AㅁHAHHVAD) and six variants of type 2 (VHHAHHAAD, AHHAH-

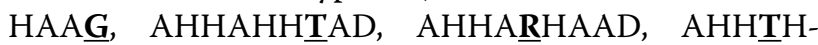
HAAD, ARHAHHAAD) were found in Myanmar $p f h r p 2$. Four variants of type 6 (VHHATD, AHHAID, DHHATD, AHHAPD) were found. Two variants of type 7 were identified, in which one variant had a deletion of alanine (-HHAAD). The frequency of each variant in Myanmar pfhrp 2 was low, ranging from 1.17 to $2.35 \%$. For Myanmar pfhrp3, an amino acid change was also observed in each type 16 (AHHAS N), type 18 (AHAAD), and type 20 (S $\underline{Y} H D D)$, but their frequencies in Myanmar pfhrp3 were also low (Table 1).

\section{Prevalence of repeat types in Myanmar pfhrp2 and pfhrp3}

Overall prevalence of each type of repeat differed in Myanmar pfhrp 2 and pfhrp3. Type 2 repeat was the most prevalent and showed high numbers of duplicates 


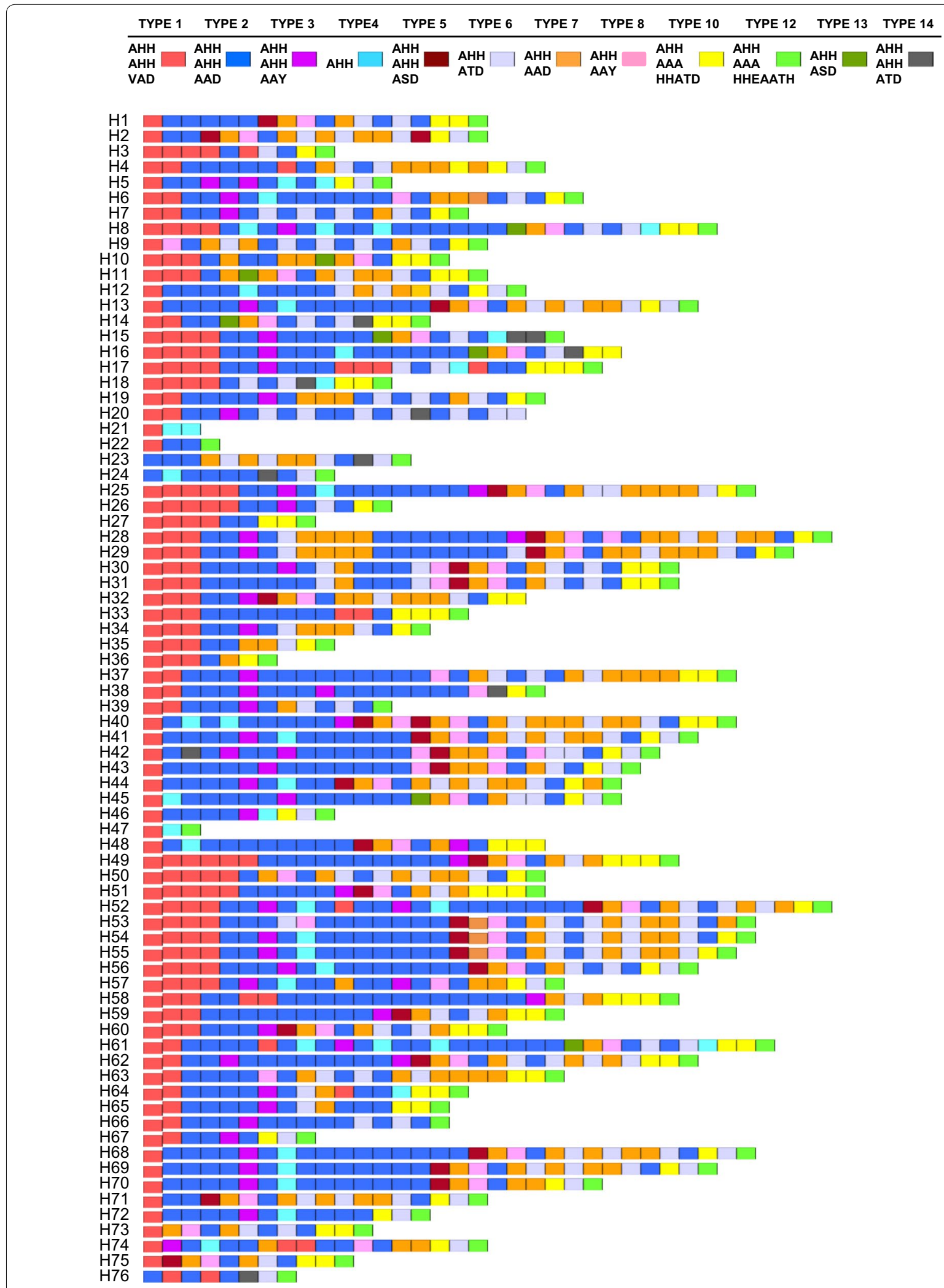

Fig. 1 Genetic variation in Myanmar pfhrp2. Seventy-six unique haplotypes were identified in Myanmar pfhrp2. The haplotypes differ in the number and organization of 12 distinct repeat types. Each repeat type is displayed as a different color 


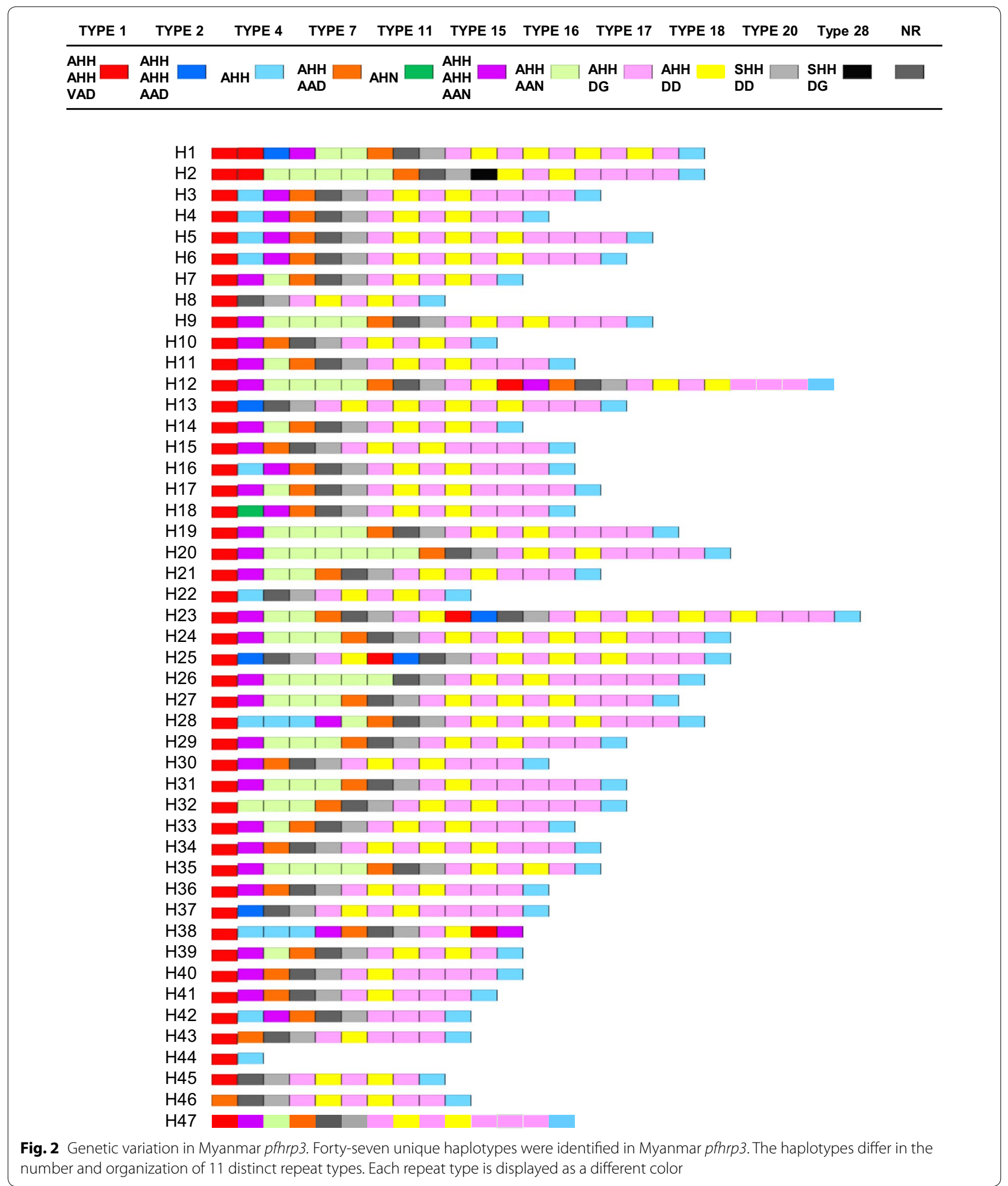

in most haplotypes of Myanmar pfhrp2. Types 1, 6 and 12 were found in most haplotypes of Myanmar pfhrp2, accounting for 96.5, 92.9 and $95.3 \%$ of sequences, respectively. Type 4, 5, and 14 repeats were observed in only a few haplotypes of Myanmar pfhrp 2 and with lower prevalence (Fig. 3). For Myanmar pfhrp3, types 1, 4, 17, 
Table 1 The amino acid changes identified in repeat types in Myanmar pfhrp2 and pfhrp3

\begin{tabular}{|c|c|c|c|}
\hline Gene & Types & Variants & Frequency (\%) \\
\hline \multirow[t]{18}{*}{ pfhrp2 } & \multirow{6}{*}{$\begin{array}{l}\text { Type } 1 \\
\text { AHHAHHVAD }\end{array}$} & AHHAHHVA $\underline{\mathbf{Y}}$ & 1.17 \\
\hline & & 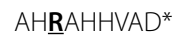 & 1.17 \\
\hline & & $\mathrm{AHHA} \underline{\mathbf{R}} \mathrm{HVAD} *$ & 1.17 \\
\hline & & AHPAAHVAD* & 1.17 \\
\hline & & AHHAHHEAD* & 1.17 \\
\hline & & $\mathrm{A} \underline{\mathbf{R}} \mathrm{HAHHVAD*}$ & 1.17 \\
\hline & \multirow{6}{*}{$\begin{array}{l}\text { Type } 2 \\
\text { AHHAHHAAD }\end{array}$} & $\underline{\mathbf{V}} \mathrm{HHAHHAAD^{* }}$ & 1.17 \\
\hline & & AHHAHHAAG & 2.35 \\
\hline & & $\mathrm{AHHAHH} \underline{\mathrm{T}} \mathrm{AD}$ & 1.17 \\
\hline & & $\mathrm{AHHA} \underline{\mathbf{R}} \mathrm{HAAD}{ }^{*}$ & 1.17 \\
\hline & & $\mathrm{AHH} \underline{\mathrm{T}} \mathrm{HHAAD}$ & 2.35 \\
\hline & & $\mathrm{A} \underline{\mathbf{R}} \mathrm{HAHHAAD}$ & 1.17 \\
\hline & \multirow{4}{*}{$\begin{array}{l}\text { Type } 6 \\
\text { AHHATD }\end{array}$} & $\underline{\mathbf{V}}$ HHATD* & 1.17 \\
\hline & & AHHAID* & 1.17 \\
\hline & & $\underline{\mathbf{D}}$ HHATD* & 1.17 \\
\hline & & $\mathrm{AHHA} \underline{\mathbf{P} D}$ & 1.17 \\
\hline & \multirow{2}{*}{$\begin{array}{l}\text { Type } 7 \\
\text { AHHAAD }\end{array}$} & $=\mathrm{HHAAD}^{*}$ & 1.17 \\
\hline & & AHHAAA $\underline{*}$ & 1.17 \\
\hline \multirow[t]{3}{*}{ pfhrp3 } & $\begin{array}{l}\text { Type } 16 \\
\text { AHHAAN }\end{array}$ & $\mathrm{AHHA} \underline{\mathrm{S}} \mathrm{N}$ & 1.78 \\
\hline & $\begin{array}{l}\text { Type } 18 \\
\text { AHHAAD }\end{array}$ & $\mathrm{A} \underline{\mathbf{R}} \mathrm{HAAD^{* }}$ & 1.78 \\
\hline & $\begin{array}{l}\text { Type } 20 \\
\text { SHHDD }\end{array}$ & $\mathrm{S} \underline{\mathbf{Y}} \mathrm{HDD}{ }^{*}$ & 1.78 \\
\hline
\end{tabular}

The amino acid changes are presented as bold. Asterisks indicate novel variants that have not been reported previously. The - in type 6 of pfhrp2 means a deletion of alanine (A) at the corresponding position. Frequency means percentage of sequences with the corresponding repeat type variant in Myanmar $p f h r p 2$ or $p f h r p 3$

18 , and 20 repeats were found commonly in most haplotypes, while the proportions of other types of repeats were variable (Fig. 3). Types 1,2 , 4, and 7 repeats were found in both Myanmar pfhrp2 and pfhrp3. Types 3, 5, $6,8,10,12,13$, and 14 were identified only in Myanmar pfhrp 2 and types $11,15,16,17,18,20$, and 28 were found only in Myanmar pfhrp3.

\section{Genetic polymorphisms of pfhrp2 and pfhrp3 in global isolates}

Comparative analysis of repeat types revealed that types $1,2,3,5,6,7,10$, and 12 were found commonly in all global pfhrp2 population (Table 2). The frequencies of types $1,2,6$, and 12 were especially high in global $p f h r p 2$. The frequencies of types $3,5,7$, and 10 differed by country. Type 8 repeat also showed high frequencies in global pfhrp2, but it was not identified in Brazil pfhrp2. Types 4,13 and 14 were observed at low frequencies in pfhrp2 from some countries, but types 15 and type 19 were found only in pfhrp 2 from China, India, Kenya, or the Solomon Islands. A similar variety of frequencies of repeat types was found in global pfhrp3 populations (Table 3). Types $1,4,7,17,18$, and 20 were detected at high frequencies in all global pfhrp3. Type 16 also showed high frequencies in all global pfhrp3, except for Myanmar. Type 15 was found in nearly all global pfhrp3 at relatively high frequencies, but it was not detected in pfhrp3 from Peru, Papua New Guinea and the Solomon Islands. Types 2, 28 and 29 were identified at low frequencies in only several countries, including Myanmar, India, Philippines, Kenya, Madagascar, Peru, or Papua New Guinea. Length variation in pfhrp 2 and pfhrp 3 was also found in the global P. falciparum population (Fig. 4). The lengths of the global pfhrp2 and pfhrp3 mainly ranged from 200-300 and 100-200 amino acids, respectively. The mean amino acid length of global pfhrp 2 was $245.2 \pm 41.9$, whereas the value for global pfhrp3 was $178.2 \pm 31.7$. Diverse length polymorphisms between and among global isolates were found in both genes. The lengths of Myanmar $p$ fhrp 2 varied from 50 to 350 amino acids (mean length $175.4 \pm 70.3$ ) and the size variation was greater than those of other countries analysed in this study. The mean lengths of $p$ fhrp 2 from East Timor (253.7 \pm 18.0$)$, Solomon Islands (260.7 \pm 23.4$)$, Honduras (262.8 \pm 14.3$)$, Brazil $(263.1 \pm 12.4)$, French Guinea (286.8 \pm 11.6$)$, Nigeria (254.0 \pm 17.3$)$, Kenya (268.4 \pm 19.7$)$, Haiti (264.4 \pm 14.4$)$, Ghana (261.3 \pm 11.0$)$, Central African Republic (258.5 \pm 14.4$)$, Vietnam 


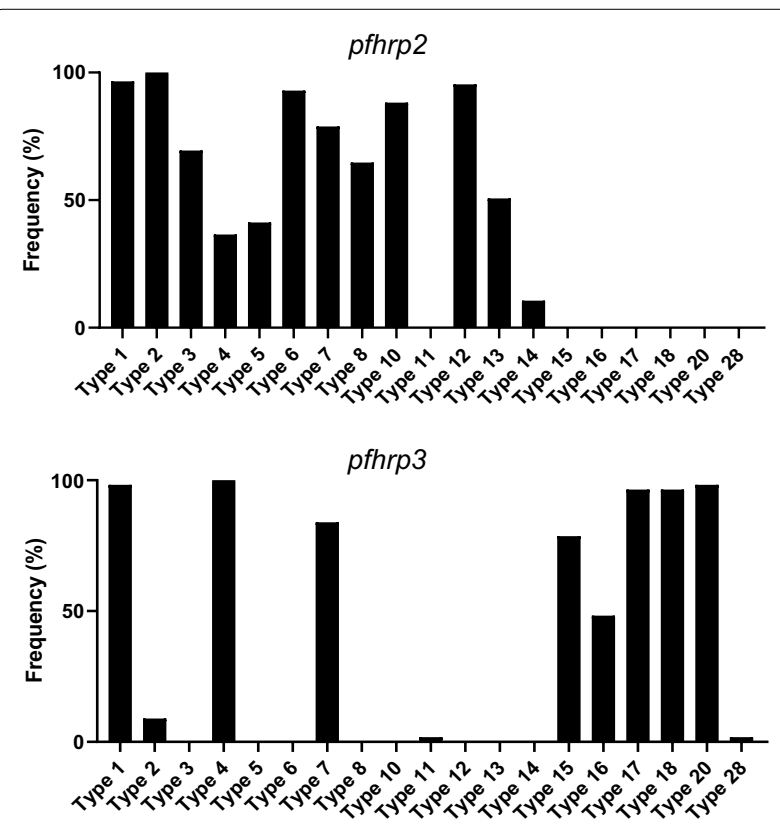

Fig. 3 Frequencies of repeat types found in Myanmar pfhrp2 and pfhrp3. The frequency of each repeat type identified in Myanmar pfhrp2 and pfhrp3 is presented

$(271.0 \pm 21.6)$, Thailand $(255.6 \pm 20.9)$, and Sri Lanka $(260.3 \pm 24.9)$ were longer than that of global pfhrp2 (245.2 \pm 41.9$)$. The mean lengths of pfhrp2 from Papua New Guinea (227.7 \pm 40.0$)$, India (240.3 \pm 24.9$)$, China-Myanmar border (212.4 \pm 56.3$)$, and Myanmar $(232.3 \pm 14.8)$ [25] were shorter than the mean length of global $p f h r p 2$. The overall lengths of Myanmar $p f h r p 3$ were remarkably shorter than global pfhrp3. Most Myanmar pfhrp 3 were about 100 amino acids in length, with a mean length of $104.7 \pm 30.4$. The mean lengths of $p$ fhrp3 from Cambodia $(268.6 \pm 27.4)$, the Solomon Islands $(168.4 \pm 29.2)$ and Papua New Guinea (163.3 \pm 21.8$)$ were shorter than that of global pfhrp3 (178.2 \pm 31.7$)$. In contrast, the mean lengths of pfhrp 3 from Colombia (211.8 \pm 21.8$)$, Peru (196.7 \pm 14.5$)$, Kenya (199.2 \pm 17.6$)$, and Philippines $(184.7 \pm 25.5)$ were longer than that of global pfhrp3.

\section{Discussion}

Malaria RDTs provide a simple, rapid and reasonably reliable diagnosis of malaria, which could aid proper treatment of the disease and offer significant benefits for malaria control and elimination. Given these advantages, global use of RDTs has been increasing rapidly in many malaria-endemic regions, and having RDTs with high specificity and sensitivity against global isolates is very important. However, genetic variation in the target antigens employed in the RDTs could affect the performance, especially their sensitivity to detect low-density malaria infections $[9,23,25]$. Understanding the genetic diversity and structure organization of the pfhrp 2 and pfhrp3 in global $P$. falciparum isolates is important because most commercially available $P$. falciparum RDTs target PfHRP2 expressed solely by the parasite [26].

This study is the first report on genetic analysis of $p f h r p 2$ and pfhrp3 in Myanmar P. falciparum isolates. Sequence analysis of Myanmar pfhrp2 and pfhrp3 suggested extensive genetic variations in the both genes consistent with previous studies for field isolates from various geographical areas $[8,9,17,22,23,25,27,28]$. Similar to previous results, overall genetic diversity was greater in pfhrp2 than $p f h r p 3$ in Myanmar isolates. Structural organizations of repeat types in Myanmar pfhrp 2 and $p f h r p 3$ were highly diverse. Most Myanmar pfhrp 2 and pfhrp3 sequences occurred only once in the analysed $P$. falciparum isolates. Characteristics shared by the isolates were also identified. The majority of Myanmar pfhrp 2 started with type 1 repeat and terminated with type 12 repeat. Similarly, the majority of Myanmar pfhrp 3 started with type 1 repeat and terminated with type 4 repeat. This conserved structural organization was also identified in global pfhrp2 and pfhrp3 [25, 27-29]; the major repeat types found in Myanmar $p f h r p 2$ and $p f h r p 3$ were also the most common repeat types observed in global $p f h r p 2$ and $p f h r p 3$. Although the frequency of each repeat type differed slightly in global isolates, repeat types $1,2,3,6,7,8$, 10 , and 12 were the most common in Myanmar and global pfhrp2 [25, 27-29]. The frequencies of repeat types 4, 5, 13, and 14 varied among global isolates. Repeat types $1,4,7,15$, $16,17,18$, and 20 were the most common in Myanmar and global pfhrp3 [25, 27-29]. Few repeat types were regional or country specific. For example, repeat types 15 and 19 were found only in limited numbers of $p$ fhrp 2 from several countries including China, India, Kenya, or the Solomon Islands. Repeat type 29 was found only in India $p f h r p 3$, and its frequency was low.

Although global pfhrp2 and pfhrp3 shared similar structural organizations, they also displayed differences. The most important differences identified were length polymorphisms, which are caused by variation in the number and arrangement of different repeat types. These length polymorphisms of global pfhrp 2 and pfhrp3 may be induced by several molecular mechanisms, such as recombination, slipped-strand mispairing event, gene conversion, and unequal crossover [30-33]. The effects of length polymorphisms in pfhrp 2 and pfhrp3 on diagnostic performance of PfHRP2-based RDTs are not clearly understood, but they could alter the binding affinity of specific monoclonal antibodies and consequently influence the sensitivity of PfHRP2-based RDTs $[9,23]$. The relationship between the combined length 


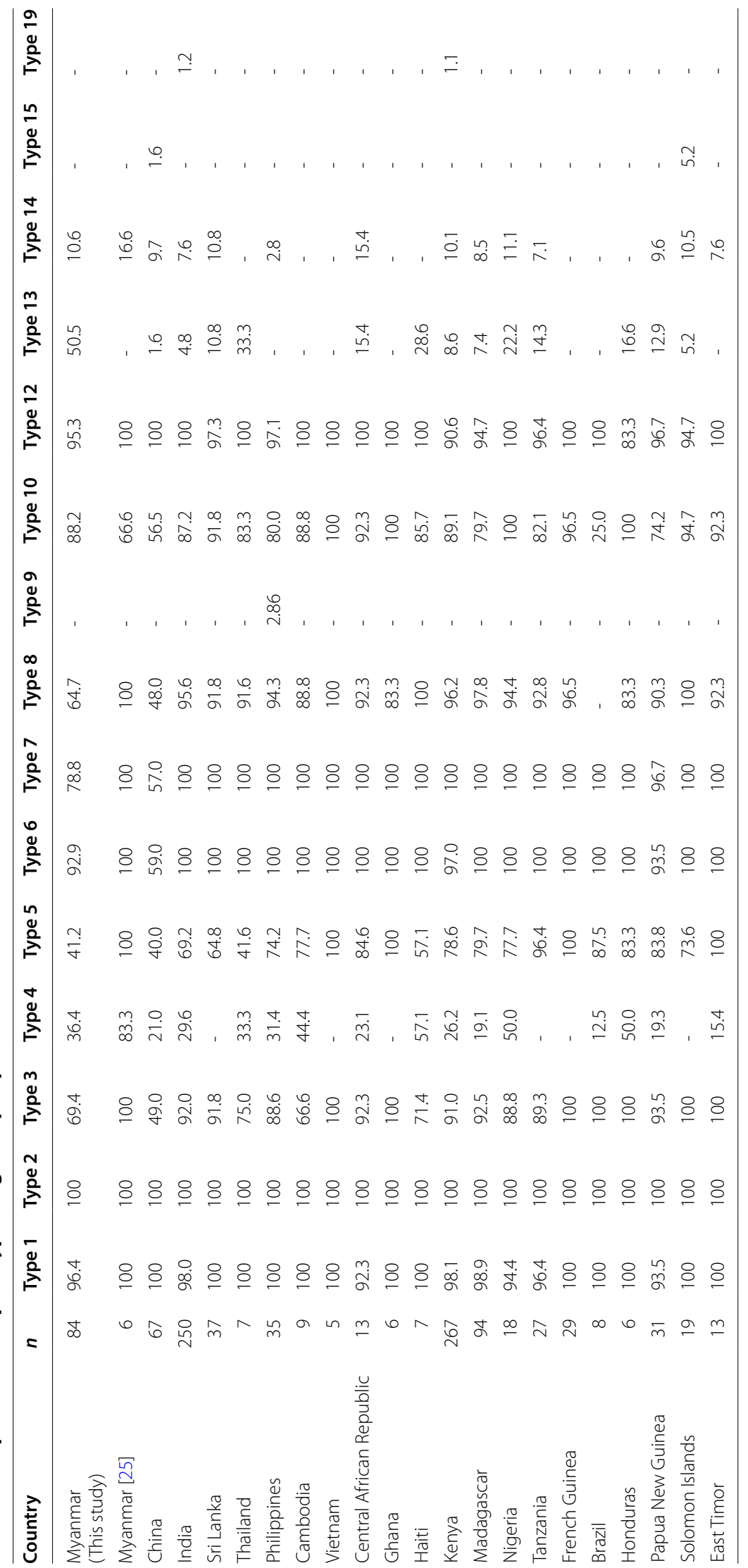


Table 3 Frequencies of repeat types in global pfhrp3

\begin{tabular}{|c|c|c|c|c|c|c|c|c|c|c|c|c|}
\hline Country & $n$ & Type 1 & Type 2 & Type 4 & Type 7 & Type 15 & Type 16 & Type 17 & Type 18 & Type 20 & Type 28 & Type 29 \\
\hline Myanmar (This study) & 56 & 97.9 & 8.9 & 100 & 85.7 & 80.3 & 57.1 & 97.9 & 97.9 & 97.9 & 1.8 & - \\
\hline Cambodia & 8 & 100 & - & 100 & 25.0 & 100 & 100 & 100 & 100 & 100 & - & - \\
\hline India & 148 & 100 & 2.0 & 100 & 100 & 99.3 & 96.6 & 100 & 98.6 & 100 & 4.1 & 2.0 \\
\hline Philippines & 7 & 100 & 28.6 & 100 & 100 & 100 & 100 & 100 & 100 & 100 & - & - \\
\hline Kenya & 270 & 98.9 & 0.4 & 100 & 100 & 98.5 & 100 & 100 & 100 & 100 & 9.3 & - \\
\hline Madagascar & 178 & 99.4 & - & 96.5 & 99.4 & 99.4 & 100 & 100 & 99.4 & 100 & 14.2 & - \\
\hline Nigeria & 16 & 100 & - & 100 & 100 & 100 & 100 & 100 & 100 & 100 & - & - \\
\hline Peru & 7 & 100 & 33.3 & 100 & 100 & - & 100 & 100 & 100 & 100 & - & - \\
\hline Colombia & 5 & 100 & - & 100 & 100 & 100 & 100 & 100 & 100 & 100 & 20.0 & - \\
\hline Papua New Guinea & 7 & 100 & - & 100 & 100 & - & 100 & 100 & 100 & 100 & - & - \\
\hline Solomon Islands & 15 & 100 & - & 100 & 100 & - & 100 & 100 & 100 & 100 & - & - \\
\hline
\end{tabular}

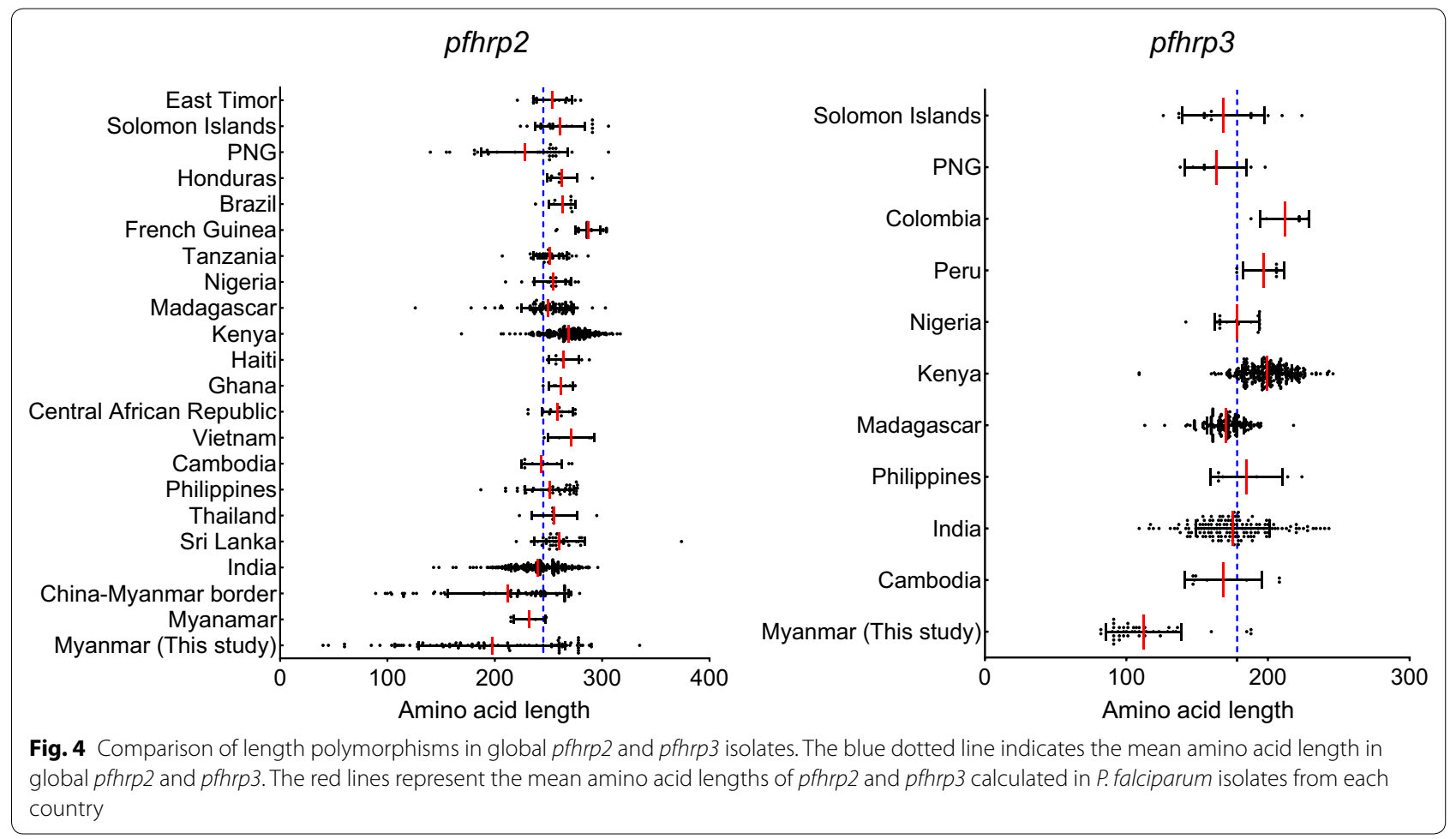

of type $2 \times$ type 7 repeats in $p f h r p 2$ and the sensitivity of PfHRP2-based RDTs has been studied previously $[8,23,25,28,34,35]$. Four studies $[8,23,34,35]$ proposed that at low parasitaemia (less than 250 parasites/ $\mu \mathrm{l})$, false-negative rates increased as combined lengths of type $2 \times$ type 7 repeats decreased. In contrast, two studies $[25,28]$ found that sensitivity of PfHRP2-RDTs was not influenced greatly by combined lengths of type $2 \times$ type 7 repeats $[25,28]$. Myanmar $p$ fhrp 2 were classified into two major groups based on the lengths of type $2 \times$ type 7 repeats, borderline group $(77.4 \%$, less than 43$)$ and group B (19\%, ranged from 50 to 100$)$. The impact of repeat length polymorphisms in Myanmar pfhrp2 on the sensitivity of RDTs was not determined in this study because RDT results for all $P$. falciparum isolates were not available. Further studies to determine the effect of pfhrp2 length polymorphisms on performance of PfHRP2-based RDTs is necessary. Amino acid changes in $p f h r p 2$ and $p f h r p 3$ are another important characteristic that cause genetic polymorphisms in global isolates $[27,36]$. In total, 17 and 3 amino acid changes were found in Myanmar pfhrp2 and pfhrp3, respectively, although the frequency of these changes was generally low. Most amino acid changes in Myanmar pfhrp2 and pfhrp3 were 
novel changes that have not been reported previously. The influence of amino acid changes identified in global pfhrp 2 and pfhrp 3 on the diagnostic performance of PfHRP2-based RDTs is also not clear yet, and therefore further study is required.

This study had several limitations. The pfhrp2 and pfhrp3 were not amplified successfully in all Myanmar $P$. falciparum isolates analysed in this study, a result explained by the poor quality of genomic DNA. Indeed, some of the $p f h r p 2$-negative samples were also negative for P. falciparum merozoite surface protein-1 (pfmsp-1) and $p f m s p-2$ amplifications. Otherwise, deletion of $p f h r p 2$ and pfhrp3 in the negative samples is also a possibility, but further study to elucidate this is necessary. Although extreme sequence variations in Myanmar pfhrp2 and $p f h r p 3$ were identified, the effect of these variations on the performance of PfHRP2-based RDTs was not elucidated clearly in this study. Further study, including larger sample sizes and RDT negative samples, is needed to understand the effect of genetic diversity and deletion of $p f h r p 2$ and pfhrp3 on the performance of the PfHRP2-RDTs.

\section{Conclusion}

Extensive genetic diversity was found in Myanmar $p$ fhrp 2 and pfhrp3. Length polymorphisms due to variation in the number and arrangement of different repeat types as well as amino acid changes contributed to the genetic diversities of Myanmar pfhrp2 and pfhrp3. Comparative sequence analysis of global $p f h r p 2$ and $p f h r p 3$ suggests that global pfhrp 2 and pfhrp3 share similar structural features, but they also differ in some features. These results may provide a better understanding of the $p f h r p 2$ and pfhrp 3 structure in global P. falciparum population and suggest useful information to develop RDTs with improved quality. Further examination of genetic diversity of $p f h r p 2$ and $p f h r p 3$ in diverse global P. falciparum populations with a larger number of isolates also is necessary to better understand the structural nature of the two genes in the global populations.

\section{Supplementary information}

Supplementary information accompanies this paper at https://doi. org/10.1186/s12936-020-03456-6.

Additional file 1: Fig. S1. Map of study site. Blood sample collection was conducted in four sites including Mandalay, Pyin Oo Lwin, Naung Cho, and Tha Beik Kyin in Upper Myanmar between 2013 and 2015.

Additional file 2: Table S1. Primers used to amplify pfhrp2 and pfhrp3.

Additional file 3: Table S2. Accession numbers of pfhrp2 sequences of global Plasmodium falciparum isolates enrolled in this study.

Additional file 4: Table S3. Accession numbers of pfhrp3 sequences of global Plasmodium falciparum isolates enrolled in this study.
Abbreviations

PCR: Polymerase chain reaction; PfHRP2: Plasmodium falciparum Histidine rich protein 2; PfLDH: Plasmodium falciparum Lactate dehydrogenase; PfHRP3: Plasmodium falciparum Histidine rich protein 3; pfmsp-1: Plasmodium falciparum Merozoite surface protein-1; pfmsp-2: Plasmodium falciparum Merozoite surface protein-2; RDT: Rapid diagnostic test; rRNA: 18 S ribosomal RNA.

\section{Acknowledgements}

We thank the staffs in the Department of Medical Research Pyin Oo Lwin Branch and the health professionals in Naung Cho and Pyin Oo Lwin townships for their contribution and technical support during blood collection. This research was supported by the National Research Foundation of Korea (NRF) grants funded by the Korean Government (NRF-2019K1A3A9A01000005 and NRF-2017M3A9B8096530).

\section{Authors' contributions}

HGL and JMK performed the genetic analysis of Myanmar pfhrp-2 and pfhrp-3. $J L, M K M, K L$ and TSK contributed to blood sample collection. HGL, JMK, JL, WGY, and BKN analysed and interpreted the data. BKN designed and supervised the experiments. HGL and BKN drafted the manuscript. All authors read and approved the final manuscript.

\section{Funding}

This research was supported by the National Research Foundation of Korea (NRF) grants funded by the Korean Government (NRF-2019K1A3A9A01000005 and NRF-2017M3A9B8096530).

\section{Availability of data and materials}

The data supporting the conclusions of this article are provided within the article and its additional files. The original datasets analysed in this current study are available from the corresponding author upon request. The nucleotide sequences reported in this study have been deposited in the GenBank database under the accession numbers KX138275-KX138311, MG417056MG417080, and MT591418-MT591439 for pfhrp2; KX138312-KX138340, and MT591440-MT591466 for pfhrp3.

\section{Ethics approval and consent to participate}

This study was approved by the Ethics Review Committee, Department of Medical Research, Myanmar (97/Ethics 2015) and by the Ethical Review Committee of Inha University School of Medicine, Korea (INHA 15-013). Informed written consent and permission were obtained from each individual.

\section{Consent for publication}

Not applicable.

\section{Competing interests}

The authors declare that they have no competing interests.

\section{Author details}

${ }^{1}$ Department of Parasitology and Tropical Medicine and Institute of Health Sciences, Gyeongsang National University College of Medicine, Jinju 52727, Republic of Korea. ${ }^{2}$ Department of Convergence Medical Science, Gyeongsang National University, Jinju 52727, Republic of Korea. ${ }^{3}$ Department of Tropical Medicine and Inha Research Institute for Medical Sciences, Inha University College of Medicine, Incheon 22212, Republic of Korea. ${ }^{4}$ Korea Mouse Phenotyping Center, Seoul National University, Seoul 08826, Republic of Korea. ${ }^{5}$ Department of Medical Research Pyin Oo Lwin Branch, Pyin Oo Lwin, Myanmar.

Received: 15 July 2020 Accepted: 18 October 2020

Published online: 02 November 2020

\section{References}

1. WHO. World malaria report 2018. Geneva: World Health Organization; 2018.

2. Moges B, Amare B, Belyhun Y, Tekeste Z, Gizachew M, Workineh M, et al. Comparison of CareStart ${ }^{\mathrm{TM}} \mathrm{HRP} 2 / \mathrm{pLDH}$ COMBO rapid malaria test with light microscopy in north-west Ethiopia. Malar J. 2012;11:234. 
3. Nyunt MH, Kyaw MP, Win KK, Myint KM, Nyunt KM. Field evaluation of HRP2 and pan pLDH-based immunochromatographic assay in therapeutic monitoring of uncomplicated falciparum malaria in Myanmar. Malar J. 2013;2:123.

4. WHO. Malaria rapid diagnostic test performance: summary results of WHO product testing of malaria RDTs: rounds 1-6 (2008-2015). Geneva: World Health Organization; 2016.

5. Moody A. Rapid diagnostic tests for malaria parasites. Clin Microbiol Rev. 2002;15:66-78.

6. Kilejian A. Homology between a histidine-rich protein from Plasmodium lophurae and a protein associated with the knob-like protrusions on membranes of erythrocytes infected with Plasmodium falciparum. J Exp Med. 1980;151:1534-8.

7. Rock EP, Marsh K, Saul AJ, Wellems TE, Taylor DW, Maloy WL, et al. Comparative analysis of the Plasmodium falciparum histidine-rich proteins HRP-I, HRP-II and HRP-III in malaria parasites of diverse origin. Parasitology. 1987;95:209-27.

8. Kumar N, Singh JP, Pande V, Mishra N, Srivastava B, Kapoor R, et al. Genetic variation in histidine rich proteins among Indian Plasmodium falciparum population: possible cause of variable sensitivity of malaria rapid diagnostic tests. Malar J. 2012;11:298.

9. Lee N, Baker J, Andrews KT, Gatton ML, Bell D, Cheng Q, et al. Effect of sequence variation in Plasmodium falciparum histidine- rich protein 2 on binding of specific monoclonal antibodies: implications for rapid diagnostic tests for malaria. J Clin Microbiol. 2006;44:2773-8.

10. Aydin-Schmidt B, Mubi M, Morris U, Petzold M, Ngasala BE, Premji Z, et al. Usefulness of Plasmodium falciparum-specific rapid diagnostic tests for assessment of parasite clearance and detection of recurrent infections after artemisinin-based combination therapy. Malar J. 2013;12:349.

11. Mehlotra RK, Howes RE, Cramer EY, Tedrow RE, Rakotomanga TA, Ramboarina S, et al. Plasmodium falciparum parasitemia and band sensitivity of the SD Bioline Malaria Ag P.f/Pan rapid diagnostic test in Madagascar. Am J Trop Med Hyg. 2019;100:1196-1.

12. Wellems TE, Howard RJ. Homologous genes encode two distinct, histidine rich proteins in a cloned isolate of Plasmodium falciparum. Proc Natl Acad Sci USA. 1986;83:6065-9.

13. Sharma YD. Genomic organization, structure and possible function of histidine rich proteins of malaria parasites. Int J Biochem. 1988:20:471-7.

14. Lee N, Gatton ML, Pelecanos A, Bubb M, Gonzalez I, Bell D, et al. Identification of optimal epitopes for Plasmodium falciparum rapid diagnostic tests that target histidine-rich proteins 2 and 3. J Clin Microbiol. 2012;50:1397-405.

15. Gupta H, Matambisso G, Galatas B, Cisteró P, Nhamussua L, Simone W, et al. Molecular surveillance of pfhrp2 and pfhrp3 deletions in Plasmodium falciparum isolates from Mozambique. Malar J. 2017;16:416.

16. Fontecha G, Mejía RE, Banegas E, Ade MP, Mendoza L, Ortiz B, et al. Deletions of pfhrp2 and pfhrp3 genes of Plasmodium falciparum from Honduras. Guatemala Nicaragua Malar J. 2018;17:320.

17. Gendrot M, Fawaz R, Dormoi J, Madamet M, Pradines B, Gendrot M, et al. Genetic diversity and deletion of Plasmodium falciparum histidine-rich protein 2 and 3: a threat to diagnosis of P. falciparum malaria. Clin Microbiol Infect. 2019;25:580-5.

18. Thomson R, Beshir KB, Cunningham J, Baiden F, Bharmal J, Bruxvoort KJ, et al. pfhrp2 and pfhrp3 gene deletions that affect malaria rapid diagnostic tests for Plasmodium falciparum: analysis of archived blood samples from 3 african countries. J Infect Dis. 2019;2209:1444-52.

19. Berzosa P, González V, Taravillo L, Mayor A, Romay-Barja M, García L, et al. First evidence of the deletion in the pfhrp2 and pfhrp3 genes in Plasmodium falciparum from Equatorial Guinea. Malar J. 2020;19:99.

20. Kojom LP, Singh V. Prevalence of Plasmodium falciparum field isolates with deletions in histidine-rich protein 2 and 3 genes in context with subSaharan Africa and India: a systematic review and meta-analysis. Malar 」. 2020;19:46.
21. Houze S, Hubert V, Le Pessec G, Le Bras J, Clain J. Combined deletions of pfhrp2 and pfhrp 3 genes result in Plasmodium falciparum malaria falsenegative rapid diagnostic test. J Clin Microbiol. 2011;49:2694-6.

22. Wurtz N, Fall B, Bui K, Pascual A, Fall M, Camara C, et al. Pfhrp2 and pfhrp3 polymorphisms in Plasmodium falciparum isolates from Dakar, Senegal: impact on rapid malaria diagnostic tests. Malar J. 2013;12:34.

23. Baker J, McCarthy J, Gatton M, Kyle DE, Belizario V, Luchavez J, et al. Genetic diversity of Plasmodium falciparum histidine-rich protein 2 (Pfhrp2) and its effect on the performance of Pfhrp2-based rapid diagnostic tests. J Infect Dis. 2005;192:870-7.

24. Kang JM, Cho PY, Moe M, Lee J, Jun H, Lee HW, et al. Comparison of the diagnostic performance of microscopic examination with nested polymerase chain reaction for optimum malaria diagnosis in Upper Myanmar. Malar J. 2017;16:119.

25. Baker J, Ho MF, Pelecanos A, Gatton M, Chen N, Abdullah S, et al. Global sequence variation in the histidine-rich proteins 2 and 3 of Plasmodium falciparum: implications for the performance of malaria rapid diagnostic tests. Malar J. 2010;9:129.

26. Mussa A, Talib M, Mohamed Z, Hajissa K. Genetic diversity of Plasmodium falciparum histidine-rich protein 2 (Pfhrp2) and its effect on the performance of Pfhrp2-based rapid diagnostic tests. BMC Res Notes. 2019:12:334.

27. Nderu D, Kimani F, Thiong'o K, Karanja E, Akinyi M, Too E, et al. Plasmodium falciparum histidine-rich protein (Pfhrp2 and 3) diversity in Western and Coastal Kenya. Sci Rep. 2019;9:1709.

28. Kumar Bharti P, Singh Chandel H, Krishna S, Nema S, Ahmad A, Udhaya-

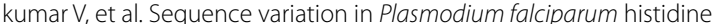
rich proteins 2 and 3 in indian isolates: implications for malaria rapid diagnostic test performance. Sci Rep. 2017;7:1308.

29. Funwei R, Nderu D, Nguetse CN, Thomas BN, Falade CO, Velavan TP, et al. Molecular surveillance of pfhrp2 and pfhrp3 genes deletion in Plasmodium falciparum isolates and the implications for rapid diagnostic tests in Nigeria. Acta Trop. 2019:196:121-5.

30. Kemp DJ, Coppel RL, Anders RF. Repetitive proteins and genes of malaria. Annu Rev Microbiol. 1987:41:181-8.

31. Smith GP. Evolution of repeated DNA sequences by unequal crossover. Science. 1976;191:528-35.

32. Levinson G, Gutman GA. Slipped-strand mispairing: A major mechanism for DNA sequence evolution. Mol Biol Evol. 1987:4:203-21.

33. Dodin G, Levoir P. Replication slippage and the dynamics of the immune response in malaria: a formal model for immunity. Parasitology. 2005:131:727-35.

34. Kumar N, Pande V, Bhatt RM, Shah NK, Mishra N, Srivastava B, et al. Genetic deletion of HRP2 and HRP3 in Indian Plasmodium falciparum population and false negative malaria rapid diagnostic test. Acta Trop. 2013;125:119-21.

35. Mariette N, Barnadas C, Bouchier C, Tichit M, Ménard D. Country-wide assessment of the genetic polymorphism in Plasmodium falciparum and Plasmodium vivax antigens detected with rapid diagnostic tests for malaria. Malar J. 2008;7:219

36. Li P, Xing H, Zhao Z, Yang Z, Cao Y, Li W, et al. Genetic diversity of Plasmodium falciparum histidine-rich protein 2 in the China-Myanmar border area. Acta Trop. 2015;152:26-31.

\section{Publisher's Note}

Springer Nature remains neutral with regard to jurisdictional claims in published maps and institutional affiliations. 